\title{
ASPERSIONES DE INSECTICIDAS EN CAMPO PARA CONTROLAR LA ESCAMA BLANCA DEL MANGO (HEMIPTERA: DIASPIDIDAE)
}

\author{
FIELD INSECTICIDE SPRAYS FOR CONTROLLING THE WHITE MANGO \\ SCALE (HEMIPTERA: DIASPIDIDAE)
}

\author{
Mario A. Urías-López ${ }^{\star}$, Luis M. Hernández-Fuentes, Jorge A. Osuna-García, M. Hilda Pérez-Barraza, \\ N. Carolina García-Álvarez y Jesús A. González-Carrillo
}

Campo Experimental Santiago Ixcuintla, Instituto Nacional de Investigaciones Forestales, Agrícolas y Pecuarias. Km 6 Carr. México-Nogales. Apdo. Postal 100. 63300, Santiago Ixcuintla, Nayarit. Tel. y Fax 01 (323) 235-0710 Ext. 125.

*Autor para correspondencia (urias.marioalfonso@inifap.gob.mx)

\section{RESUMEN}

Ante la amplia distribución y los daños que produce en Nayarit la escama blanca del mango (EBM) Aulacaspis tubercularis Newstead, se establecieron seis experimentos en condiciones de campo durante los años de 2004 a 2007. El objetivo fue evaluar la eficacia biológica de productos con propiedades insecticidas contra la EBM, como posibles opciones para el control de la plaga. Los estudios se realizaron en huertos comerciales de mango 'Ataulfo' y 'Haden' situados en Las Varas y Atonalisco, Nayarit. Se evaluaron varias dosis de cuatro insecticidas sintéticos, dos detergentes comerciales, aceite mineral (citrolina), mezclas de citrolina más malatión y un concentrado comercial de ajo. Las variables fueron: número de hembras, colonias de machos y total de individuos por hoja. Con las mezclas de citrolina $\left(50 \mathrm{~mL} \mathrm{~L}^{-1}\right)+$ malatión (1.11 mL L $\mathrm{mL}^{-1}$ o $\left.2.22 \mathrm{~mL} \mathrm{~L}^{-1}\right)$, se obtuvo hasta $98.3 \%$ y $100 \%$ de control de escamas, respectivamente. Las aspersiones de piriproxifeno $\left(0.3 \mathrm{~mL} \mathrm{~L}^{-1}\right)$ o dimetoato $\left(2.0 \mathrm{~mL} \mathrm{~L}^{-1}\right)$, controlaron el $100 \%$ de la EBM. Con el aceite mineral (citrolina) aplicado a dosis de $50 \mathrm{~mL} \mathrm{~L}^{-1}$ o $75 \mathrm{~mL}$ $\mathrm{L}^{-1}$, el máximo control de escamas fue $91.3 \%$ y $97.5 \%$, respectivamente. Con los detergentes comerciales Roma ${ }^{\circledR}$ y Ariel ${ }^{\circledR}\left(20 \mathrm{~g} \mathrm{~L}^{-1}\right)$ se lograron los mejores controles durante la primera semana con $75.5 \%$ y $55.3 \%$, respectivamente. Los resultados con cipermetrina a 0.24 y $0.36 \mathrm{~mL} \mathrm{~L}^{-1}$, $\mathrm{y}$ con el producto a base de ajo fueron inconsistentes.

Palabras clave: Aulacaspis tubercularis, Mangifera indica, aceite mineral, detergentes, control de plagas.

\section{SUMMARY}

Considering the wide distribution and damage caused by white mango scale (WMS) Aulacaspis tubercularis Newstead in Nayarit, México, six field insecticide trials were conducted from 2004 to 2007 for the control of the pest. The main objective was to determine efficacy of selected insecticides on the WMS. Experiments were conducted with 'Ataulfo' and 'Haden' cultivars in mango orchards in Las Varas, and Atonalisco, Nayarit. The main parameters were number of females, male colonies and total scales per leaf. Four synthetic insecticides, two laundry detergents, mineral oil (citroline), mixtures of mineral oil + malathion and a commercial garlic extract were evaluated. The mixtures of mineral oil $\left(50 \mathrm{~mL} \mathrm{~L}^{-1}\right)+$ malathion $\left(1.11 \mathrm{~mL} \mathrm{~L}^{-1}\right.$ or $2.22 \mathrm{~mL}$ $\left.\mathrm{L}^{-1}\right)$, were controlled effectively $98.3 \%$ y $100 \%$ of scales, respectively. Pyriproxyfen $\left(0.3 \mathrm{~mL} \mathrm{~L}^{-1}\right)$ or dimetoate $\left(2.0 \mathrm{~mL} \mathrm{~L}^{-1}\right)$ recorded a $100 \%$ control. Mineral oil $\left(50 \mathrm{~mL} \mathrm{~L}^{-1}\right.$ or $\left.75 \mathrm{~mL} \mathrm{~L}^{-1}\right)$ was effective controlling $91.3 \%$ and $97.5 \%$ of the scales, respectively. Commercial brand Roma ${ }^{\circledR}$ and Ariel ${ }^{\circledR}$ detergents at $20 \mathrm{~g} \mathrm{~L}^{-1}$, sprayed on foliage controlled
$75.5 \%$ y $55.3 \%$ of scales, respectively. Control of the WMS with garlic extract or cipermetrine $\left(0.24\right.$ or $\left.0.36 \mathrm{~mL} \mathrm{~L}^{-1}\right)$ was inconsistent.

Index words: Aulacaspis tubercularis, Mangifera indica, mineral oil, detergents, pest control.

\section{INTRODUCCIÓN}

El mango (Mangifera indica L.) es el frutal más importante de Nayarit, México por la superficie que se cultiva y empleos directos e indirectos que genera. En Nayarit la superficie establecida es cercana a 22000 ha con una producción aproximada a $230000 \mathrm{t}$ (SAGARPA-CESAVENAY, 2007). El cultivo de mango presenta problemas fitosanitarios relativamente recientes, como la escama blanca del mango (EBM) Aulacaspis tubercularis Newstead (Hemiptera: Diaspididae), la cochinilla rosada del hibisco Maconellicoccus hirsuts Green y el complejo de trips (Urías, 2006). En Nayarit, la EBM es una plaga importante del cultivo ya que está establecida en más de 10000 ha y si no se le controla puede reducir hasta en $50 \%$ el volumen de la cosecha (Urías, 2006). Los municipios de la entidad con más alta infestación son Compostela, San Blas y Tepic, con promedio superiores de 4.39 hembras y colonias por hoja (GarcíaÁlvarez et al., 2008). Los promedios más altos de escamas por huerto se han registrado en las localidades Alta Vista, La Lima y el Divisadero del municipio de Compostela, con 10.0, 9.7 y 7.1 hembras y colonias por hoja, respectivamente (García-Álvarez et al., 2008).

Las hembras y colonias de machos se localizan en hojas, tallos tiernos y frutos (Urías, 2006; Urías-López et al., 2010), como se ilustra en la Figura 1. En Ecuador, el ciclo biológico de la hembra de A. tubercularis es de $52 \mathrm{~d}$, y $36 \mathrm{~d}$ en el macho; las poblaciones más altas de esta especie ocurren de octubre a enero (Arias et al., 2004). En Nayarit la EBM pasa por tres fases de desarrollo poblacional: la primera fase es de baja población, de agosto a noviembre; en la segunda ocurre un crecimiento poblacional de diciembre a febrero. 
En la tercera fase ocurren los niveles más altos de la población, de marzo hasta inicio de lluvias en julio (Urías-López y Flores-Canales, 2005; Urías-López et al., 2010).

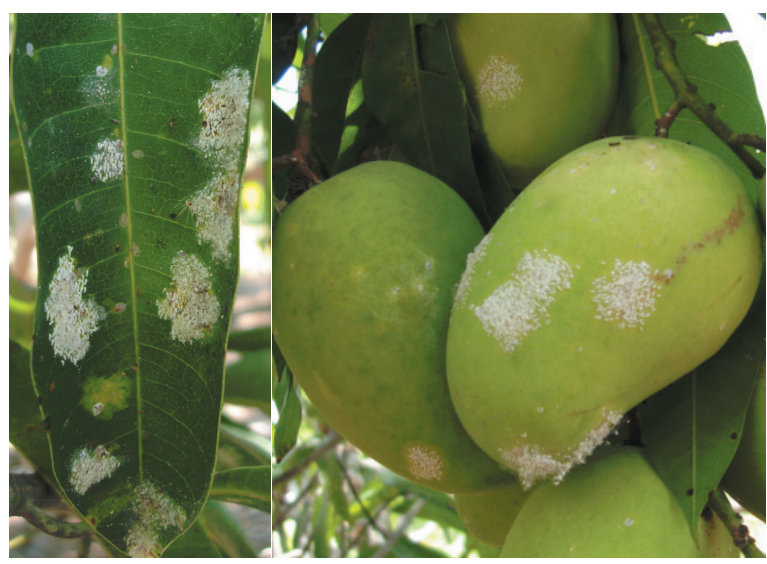

Figura 1. Hoja y frutos infestados con escama blanca.

Existe escasa información del control químico de la EBM, aunque en otras especies de escamas este tipo de control es una opción utilizada (Thakur y Hameed, 1981). Rebek y Clifford (2003) lograron el mejor control de la escama Unaspis euonymi (Comstock) con una baja afectación de su parasitoide Encarsia citrina (Crawford) mediante la aplicación al suelo de acefato, o por medio de su aplicación foliar con el regulador de crecimiento piriproxifeno. Este mismo producto mostró alta efectividad al controlar en condiciones de campo al cóccido Ceroplastes destructor Newstead (Wakgari y Giliomee, 2001). En el caso de A. tubercularis, sin indicar ingrediente activo, Arias et al. (2004) obtuvieron buen control con los insecticidas comerciales Cochibiol ${ }^{\circledR}$, Banaoil ${ }^{\circledR}$ y aceite agrícola, mientras que Le Lagadec et al. (2009) también lograron resultados promisorios con el insecticida neo-nicotenoide, tiametoxam.

En Nayarit existen varias especies depredadoras de la EBM (González-Carrillo et al., 2008) que deberían ser protegidas durante el control químico de la escama, pero se desconocen productos que sean efectivos contra la plaga y simultáneamente sean de baja toxicidad contra los insectos benéficos. Los detergentes, jabones, aceites naturales o derivados de petróleo podrían ser buenas alternativas para el control de la EBM, con bajo riesgo para la fauna benefactora, pero se desconocen resultados de investigaciones de este tipo que se hayan hecho en mango. Al respecto, en China algunos aceites derivados del petróleo han resultado efectivos en el control de las ninfas de D. citri en condiciones de campo (Rae et al., 1997). Además, mediante la aplicación de aceite derivado del petróleo $(2.5 \%)$ o con aceite de soya (Glycine max L.) (5.0\%), en evaluaciones de campo se eliminó 85 \% y 98 \% de la escama de San José, Quadraspidiotus perniciosus (Comstock), respectivamente (Pless et al., 1995). En teoría, los aceites obstruyen los espiráculos
(Smith y Pearce, 1948), o producen desecación y ablandamiento de la cutícula, lo que provoca deshidratación y sofocación (Stadler y Buteller, 2009). La información sobre el uso de detergentes para el control de plagas también es escasa, algunos pueden tener efecto acaricida al lograr una mortalidad hasta de 91.3 \% de Panonychus citri (Curkovic y Araya 2004), y con algunos jabones y detergentes se han logrado controles hasta de $85.0 \%$ en Bemisia tabaci (Butler et al., 1993).

Debido a que se carece de información sobre el control químico de la EBM y a que los productores de mango en Nayarit aplican diversos tipos de insecticidas sintéticos sin evidencia técnica de su efectividad, el objetivo de este estudio fue evaluar la eficacia de productos con propiedades insecticidas contra la EBM, así como otros productos que teóricamente puedan utilizarse con relativa seguridad para el control de la plaga.

\section{MATERIALES Y MÉTODOS}

De 2004 a 2007 se establecieron seis experimentos en tres huertos comerciales de mango 'Ataulfo' y tres de 'Haden' situados en Atonalisco y Las Varas, Nay., respectivamente (Cuadro 1). Se seleccionaron árboles de porte bajo a mediano (entre 4 y $6 \mathrm{~m}$ de altura) y vigor uniforme, establecidos a distancias de $10 \times 10 \mathrm{~m}$ entre hilera y árboles. Los tratamientos (productos) (Cuadro 1) se empezaron a aplicar después de la floración y del cuajado de fruto (abril), hasta la maduración de los mismos (junio), periodo que coincide con alta población de escamas en esas localidades. Se utilizó un aspersor de mochila motorizado y se aplicó con gota muy fina para lograr una cobertura uniforme de los productos. En cada caso los productos se mezclaron en 10 $\mathrm{L}$ de agua y se asperjaron en cinco árboles (cada árbol fue una repetición) por cada tratamiento, lo que equivale a un gasto de $200 \mathrm{~L}$ de agua en 100 árboles (número aproximado de árboles/ha en huertos comerciales). En la aplicación de la citrolina y la mezcla de citrolina + malatión, se utilizó un dispersante comercial (Surfacid $\AA$ ) en dosis de $5 \mathrm{~mL} \mathrm{~L}^{-1}$ de agua (1.0 L/100 árboles).

Algunos productos que se incluyeron en esta investigación son de uso común entre los productores, y los otros porque teóricamente tendrían menor impacto negativo sobre los depredadores de la escama. Por carecer de información científica sobre dosis, se aplicaron las recomendadas por los fabricantes.

\section{Variables registradas}

Para evaluar la eficacia de los productos, se registró la presencia de escamas en el follaje un día antes de la aplicación y luego cada semana durante dos semanas (Experimentos 3, 
Cuadro 1. Características de los huertos y de los tratamientos aplicados en seis experimentos.

\begin{tabular}{|c|c|c|c|c|}
\hline Experimento & Localidad-Cultivar & Fecha de Inicio $^{\dagger}$ & Producto & Dosis $^{\dagger \dagger}$ \\
\hline \multirow[t]{2}{*}{1} & Las Varas- 'Haden' & 21/Abr-04 & Citrolina + malatión & $50 \mathrm{~mL} \mathrm{~L}^{-1}+2.22 \mathrm{~mL} \mathrm{~L}^{-1}$ \\
\hline & & & Concentrado de ajo & $4.6 \mathrm{~mL} \mathrm{~L}^{-1}$ \\
\hline \multirow[t]{3}{*}{2} & Las Varas- 'Haden' & 14/Jun/04 & Fertilizante líquido ${ }^{\dagger \dagger \dagger}$ & $20 \mathrm{~mL} \mathrm{~L}^{-1}$ \\
\hline & & & Piriproxifeno & $0.3 \mathrm{~mL} \mathrm{~L}^{-1}$ \\
\hline & & & Dimetoato & $2.0 \mathrm{~mL} \mathrm{~L}^{-1}$ \\
\hline \multirow[t]{3}{*}{3} & Las Varas- 'Haden' & 19/May/05 & Citrolina & $50 \mathrm{~mL} \mathrm{~L}^{-1}$ \\
\hline & & & Citrolina + malatión & $25 \mathrm{~mL} \mathrm{~L}^{-1}+1.11 \mathrm{~mL} \mathrm{~L}^{-1}$ \\
\hline & & & Cipermetrina & $0.36 \mathrm{~mL} \mathrm{~L}^{-1}$ \\
\hline \multirow[t]{3}{*}{4} & Las Varas-'Ataulfo' & 19/Jun/05 & Citrolina & $50 \mathrm{~mL} \mathrm{~L}^{-1}$ \\
\hline & & & Citrolina + malatión & $25 \mathrm{~mL} \mathrm{~L}^{-1}+1.11 \mathrm{~mL} \mathrm{~L}^{-1}$ \\
\hline & & & Cipermetrina & $0.24 \mathrm{~mL} \mathrm{~L}^{-1}$ \\
\hline \multirow[t]{3}{*}{5} & Atonalisco-'Ataulfo' & 26/May/06 & Citrolina & $75 \mathrm{~mL} \mathrm{~L}^{-1}$ \\
\hline & & & Citrolina + malatión & $50 \mathrm{~mL} \mathrm{~L}^{-1}+1.11 \mathrm{~mL} \mathrm{~L}^{-1}$ \\
\hline & & & Citrolina + malatión & $50 \mathrm{~mL} \mathrm{~L}^{-1}+2.22 \mathrm{~mL} \mathrm{~L}^{-1}$ \\
\hline \multirow[t]{3}{*}{6} & Atonalisco-'Ataulfo' & 29-May/07 & Detergente Ariel ${ }^{\circledR}$ & $20 \mathrm{~g} \mathrm{~L}^{-1}$ \\
\hline & & & Detergente Roma ${ }^{\circledR}$ & $20 \mathrm{~g} \mathrm{~L}^{-1}$ \\
\hline & & & Citrolina & $50 \mathrm{~mL} \mathrm{~L}^{-1}$ \\
\hline
\end{tabular}

${ }^{\dagger}$ Es la fecha de inicio de los tratamientos; ${ }^{\dagger \dagger}$ Dosis de ingrediente activo, por litro de agua; ${ }^{\dagger \dagger}$ Dosis de producto comercial, ya que los productores de mango en la región usan el fertilizante líquido y dimetoato como insecticidas. En cada experimento se incluyó un tratamiento testigo sin aplicación.

4 y 5), tres semanas (Experimentos 2 y 6) y cuatro semanas (Experimento 1). En los experimentos 3, 4 y 5 no se hicieron muestreos posteriores a las dos semanas porque la presencia de lluvias interfirió con la población de escamas. Para estimar la población de la plaga se consideró el número de hembras y el de las colonias de machos; con estas dos variables se calculó el total de escamas (hembras y colonias). En los muestreos se aplicó la metodología de campo utilizada por Urías-López et al. (2010), que consiste en seleccionar al azar cinco árboles por tratamiento, en los que se marcan cuatro ramas por árbol, una en cada punto cardinal, y de cada rama se cortan cuatro hojas situadas en el penúltimo flujo vegetativo (16 hojas por árbol). Las hojas elegidas se etiquetaron y se transportaron al laboratorio para contar los ejemplares vivos con un microscopio estereoscópico marca Zeiss ${ }^{\circledR}(\bmod$. Stemi DV4).

\section{Análisis estadístico}

Se hicieron análisis de varianza individuales por localidad con base en un diseño de bloques al azar y cinco repeticiones (árboles), con el paquete SAS (SAS Institute, 2000). La comparación de medias fue mediante la prueba de Tukey ( $\mathrm{P}$ $\leq 0.05)$, y además se calcularon sus correspondientes errores estándar $( \pm \mathrm{EE})$. Las variables se analizaron con datos originales y con datos transformados (logaritmo base diez +1 ); como no hubo diferencia con ambos procedimientos, los resultados se presentan con datos no transformados.

\section{RESULTADOS Y DISCUSIÓN}

\section{Experimento 1}

Este experimento efectuado con el cultivar 'Haden' fue en el que los tratamientos se aplicaron más temprano durante el año y quizá por esta razón las poblaciones de la plaga fueron bajas; en etapas tardías (i. e., como en los demás experimentos), las poblaciones son normalmente más altas (Urías et al., 2010). Antes de la aplicación de los productos y hasta tres semanas después de aplicados, el número de colonias por hoja fue similar al testigo, pero a la cuarta semana, el número de colonias fue menor que en el testigo.

En particular en esta semana, en los árboles tratados con malatión más citrolina no hubo colonias, y en los tratados con el producto a base de ajo (Allium sativum) hubo 0.06 colonias por hoja, que equivalen a un control de $57 \%$ con respecto al testigo, valor que se considera bajo (Cuadro 2).

Respecto al total de estados biológicos de la EBM, la mezcla de malatión + citrolina controló por completo el total de escamas durante todo el periodo de evaluación, mientras que el producto a base de ajo aún presentó escamas pero con valores totales menores que en el testigo, hasta la tercera semana de haberse aplicado (Cuadro 2). De este ensayo se concluye que la mezcla de citrolina $\left(50 \mathrm{~mL} \mathrm{~L}^{-1}\right)+$ malatión (2.2 $\left.\mathrm{mL} \mathrm{L}^{-1}\right)$ controló por completo a las escamas; los 
Cuadro 2. Control químico de la escama blanca del mango en árboles ddel cultivar 'Haden'. Experimento 1. Las Varas, Nayarit. 2004.

\begin{tabular}{|c|c|c|c|c|}
\hline \multirow{2}{*}{ Tratamiento } & \multicolumn{4}{|c|}{ Insectos por hoja ( \pm Error estándar) } \\
\hline & Semana 0 & Semana $1^{\dagger}$ & Semana 3 & Semana 4 \\
\hline & \multicolumn{4}{|c|}{ Colonias por hoja } \\
\hline Control & $0.25(0.07) \mathrm{a}$ & $0.01(0.02) \mathrm{a}$ & $0.04(0.01) \mathrm{a}$ & $0.14(0.03) \mathrm{a}$ \\
\hline Concentrado de ajo & $0.24(0.07) \mathrm{a}$ & $0.04(0.02) \mathrm{a}$ & $0.01(0.01) \mathrm{a}$ & $0.06(0.03) b$ \\
\hline \multirow[t]{2}{*}{ Malatión + citrolina } & $0.20(0.07) \mathrm{a}$ & $0.00(0.02) \mathrm{a}$ & $0.00(0.01) \mathrm{a}$ & $0.00(0.03) \mathrm{b}$ \\
\hline & \multicolumn{4}{|c|}{ Total por hoja } \\
\hline Control & $0.75(0.2) \mathrm{a}$ & $0.24(0.23) \mathrm{a}$ & $0.13(0.04) \mathrm{a}$ & $0.65(0.15) \mathrm{a}$ \\
\hline Concentrado de ajo & $0.71(0.2) \mathrm{a}$ & $0.20(0.23) \mathrm{a}$ & $0.01(0.04) \mathrm{b}$ & $0.21(0.15) \mathrm{b}$ \\
\hline Malatión + citrolina & $0.78(0.2) \mathrm{a}$ & $0.00(0.23) b$ & $0.00(0.04) b$ & $0.00(0.15) \mathrm{b}$ \\
\hline
\end{tabular}

resultados con el producto a base de ajo fueron variables y de menor magnitud, pero alcanzaron un control máximo de $92.0 \%$ del total de escamas.

\section{Experimento 2}

Antes de la aplicación de los productos la diferencia en el promedio de colonias y del total de escamas entre tratamientos fue mínima. En las tres fechas de evaluación, se detectó diferencia significativa de los productos aplicados con respecto al testigo, en el número de colonias de EMB y en el total de individuos por hoja. En la segunda y tercera semanas después de la aplicación, los tres productos lograron el control total o cercano a 100 \% (Cuadro 3).

En este experimento las aspersiones al follaje del fertilizante foliar (a $\left.20 \mathrm{~mL} \mathrm{~L}^{-1}\right)$, de piriproxifeno $\left(0.3 \mathrm{~mL} \mathrm{~L}^{-1}\right)$ o dimetoato $\left(2.0 \mathrm{~mL} \mathrm{~L}^{-1}\right)$, lograron controlar hasta $100 \%$ de la EBM. Este resultado es importante porque no se tenían datos del efecto de estos productos contra esta plaga, y ahora constituyen una opción para su control. Sin embargo, debería considerarse que el dimetoato puede tener efectos negativo sobre insectos benéficos, y que el piriproxifeno (insecticida regulador de crecimiento), que teóricamente debe tener menor impacto negativo en ese sentido, es un producto caro.

\section{Experimento 3}

En el muestreo anterior a la aplicación de los productos, el promedio de colonias y el total de escamas fue más alto en los árboles en los que se usaría cipermetrina. Una semana después de la aplicación, las colonias de escamas por hoja en los árboles tratados con los tres productos fueron menos numerosas que en el testigo, y dos semanas después de la aplicación el menor número de colonias por hoja ocu- rrió en los árboles tratados con la mezcla de malatión + citrolina (Cuadro 4).

Respecto al total de individuos, una semana después de la aplicación las cifras de individuos registrados en los árboles tratados fueron más bajos que en el testigo (Cuadro 4); en la segunda semana el promedio más bajo se obtuvo con la mezcla de citrolina + malatión ( 0.11 total de escamas por hoja). Los resultados de este ensayo muestran que el mejor control con cipermetrina $(65.8 \%)$ fue solo hasta la primera semana. En cambio, con la mezcla de citrolina (25 $\left.\mathrm{mL} \mathrm{L}^{-1}\right)+$ malatión $\left(1.11 \mathrm{~mL} \mathrm{~L}^{-1}\right)$ se logró un buen control (93.4\%) durante las primeras dos semanas posteriores a la aplicación.

\section{Experimento 4}

Antes de la aplicación de productos, aunque en el testigo se registraron los mayores valores tanto de colonias como del total de individuos de EMB por hoja, estos valores se incrementaron a casi el doble durante el desarrollo del experimento, en comparación con los otros tratamientos (Cuadro 5), probablemente debido a alguna condición favorable para la plaga en este huerto y periodo de estudio. En la primera semana después de la aplicación la menor cantidad de colonias ( 0.07 colonias/hoja) y de número total de escamas (0.10 por hoja), se registró en los árboles asperjados con la mezcla de citrolina + malatión, lo cual representó $96.8 \%$ y $96.0 \%$ de control, respectivamente.

En la segunda semana el mejor tratamiento para colonias y del total de escamas también fue con la mezcla de citrolina + malatión con controles de 95.0 y $92.5 \%$, respectivamente. Los resultados de este ensayo indican que la mezcla de citrolina $\left(25 \mathrm{~mL} \mathrm{~L}^{-1}\right)+$ malatión $\left(1.11 \mathrm{~mL} \mathrm{~L}^{-1}\right)$ fue nuevamente el mejor tratamiento con 96 y $92.5 \%$ de 
Cuadro 3. Control químico de la escama blanca del mango en árboles del cultivar 'Haden'. Experimento 2. Las Varas, Nayarit. 2004.

\begin{tabular}{|c|c|c|c|c|}
\hline \multirow{2}{*}{ Tratamiento } & \multicolumn{4}{|c|}{ Insectos por hoja ( \pm Error estándar) } \\
\hline & Semana 0 & Semana 1 & Semana 2 & Semana 3 \\
\hline & \multicolumn{4}{|c|}{ Colonias por hoja } \\
\hline Control & $2.29(0.24) \mathrm{ab}$ & $1.90(0.1) \mathrm{a}$ & $1.94(0.1) \mathrm{a}$ & $0.54(0.05) \mathrm{a}$ \\
\hline Fertilizante foliar & $2.83(0.24) \mathrm{a}$ & $0.24(0.1) b$ & $0.00(0.1) b$ & $0.01(0.05) b$ \\
\hline Piriproxifeno & $2.29(0.24) \mathrm{ab}$ & $0.27(0.1) b$ & $0.00(0.1) b$ & $0.00(0.05) b$ \\
\hline \multirow[t]{2}{*}{ Dimetoato } & $2.01(0.24) b$ & $0.05(0.1) \mathrm{b}$ & $0.01(0.1) b$ & $0.00(0.05) \mathrm{b}$ \\
\hline & \multicolumn{4}{|c|}{ Total por hoja } \\
\hline Control & $4.33(0.41) \mathrm{a}$ & $3.90(0.19) \mathrm{a}$ & $3.39(0.18) \mathrm{a}$ & $1.10(0.18) \mathrm{a}$ \\
\hline Fertilizante foliar & $3.79(0.41) \mathrm{ab}$ & $0.49(0.19) \mathrm{b}$ & $0.00(0.18) b$ & $0.01(0.18) b$ \\
\hline Piriproxifeno & $3.25(0.41) \mathrm{ab}$ & $0.55(0.19) \mathrm{b}$ & $0.00(0.18) b$ & $0.00(0.18) b$ \\
\hline Dimetoato & $2.85(0.41) \mathrm{b}$ & $0.09(0.19) \mathrm{b}$ & $0.06(0.18) b$ & $0.01(0.18) b$ \\
\hline
\end{tabular}

Cuadro 4. Control químico de la escama blanca del mango en árboles del cultivar 'Haden'. Experimento 3. Las Varas, Nayarit. 2005.

\begin{tabular}{lccc}
\hline \multirow{2}{*}{ Tratamiento } & \multicolumn{3}{c}{ Insectos por hoja $( \pm$ Error estándar) } \\
\cline { 2 - 4 } Cipermetrina & Semana 0 & Semana 1 & Semana 2 \\
Control & $0.54(0.07) \mathrm{a}$ & $0.00(0.03) \mathrm{b}$ & $0.71(0.09) \mathrm{a}$ \\
Citrolina & $0.17(0.07) \mathrm{b}$ & $0.24(0.03) \mathrm{a}$ & $0.32(0.09) \mathrm{b}$ \\
Citrolina + malatión & $0.05(0.07) \mathrm{b}$ & $0.00(0.03) \mathrm{b}$ & $0.34(0.09) \mathrm{b}$ \\
& $0.00(0.07) \mathrm{b}$ & $0.02(0.03) \mathrm{b}$ & $0.06(0.09) \mathrm{c}$ \\
Control & & Total por hoja & \\
Cipermetrina & $0.86(0.16) \mathrm{b}$ & $1.20(0.12) \mathrm{a}$ & $1.67(0.19) \mathrm{a}$ \\
Citrolina & $1.58(0.16) \mathrm{a}$ & $0.41(0.12) \mathrm{b}$ & $1.47(0.19) \mathrm{a}$ \\
Citrolina + malatión & $0.49(0.16) \mathrm{bc}$ & $0.26(0.12) \mathrm{b}$ & $0.72(0.19) \mathrm{b}$ \\
\hline
\end{tabular}

Medias con la misma letra en cada columna son estadísticamente iguales (Tukey, 0.05).

control en la primera y segunda semanas, respectivamente. La aplicación de solo citrolina $\left(50 \mathrm{~mL} \mathrm{~L}^{-1}\right)$ o de cipermetrina $\left(0.24 \mathrm{~mL} \mathrm{~L}^{-1}\right)$ fue efectiva en este ensayo pero sólo hasta la primera semana de aplicación, con un control de 91.3 y $92.5 \%$ del total de escamas.

\section{Experimento 5}

En el muestro anterior a la aplicación de los productos no hubo diferencias significativas entre tratamientos con respecto al número de colonias y al total de individuos de la EBM (Cuadro 6). Una semana después se detectó diferencia significativa entre tratamientos; en el caso de las colonias, la mayor población ocurrió en los árboles testigo y la más baja en los tratados con citrolina + malatión (2.11
$\left.\mathrm{mL} \mathrm{L}^{-1}\right)$. Con este mismo tratamiento se registró la menor cantidad del total de escamas (0.16 individuos por hoja), lo que representa $81.1 \%$ de control.

A la segunda semana después de la aplicación, los promedios más altos de colonias ( 0.16 colonias/hoja) y de escamas totales (2.36 escamas/hoja) se registraron en los árboles testigo; la menor cantidad ocurrió en los tratamientos a base de citrolina + malatión o con citrolina sola (Cuadro 6). Con el tratamiento de citrolina + malatión $\left(2.5 \mathrm{~mL} \mathrm{~L}^{-1}\right)$, se logró un control de $98.7 \%$, y con citrolina sola $\left(75 \mathrm{~mL} \mathrm{~L}^{-1}\right)$ el control fue de $97.5 \%$; este último resultado es comparable al obtenido por Arias et al. (2004) con aceite agrícola. Los resultados de este experimento indican que la citrolina sola $\left(75 \mathrm{~mL} \mathrm{~L}^{-1}\right)$ o en mezclas con citrolina $\left(50 \mathrm{~mL} \mathrm{~L}^{-1}\right)$ más 
Cuadro 5. Control químico de la escama blanca del mango en árboles del cultivar 'Ataulfo'. Experimento 4. Las Varas, Nayarit. 2005.

\begin{tabular}{lccc}
\hline \multirow{2}{*}{ Tratamiento } & \multicolumn{3}{c}{ Insectos por hoja $( \pm$ Error estándar $)$} \\
\cline { 2 - 4 } Comana 0 & Cemana 1 & Semana 2 \\
Citrolina & $1.77(0.17) \mathrm{a}$ & $2.18(0.11) \mathrm{a}$ & $3.17(0.24) \mathrm{a}$ \\
Cipermetrina & $0.24(0.17) \mathrm{b}$ & $0.14(0.11) \mathrm{b}$ & $2.91(0.24) \mathrm{b}$ \\
Citrolina + malatión & $0.39(0.17) \mathrm{b}$ & $0.16(0.11) \mathrm{b}$ & $1.54(0.24) \mathrm{c}$ \\
& $0.84(0.17) \mathrm{b}$ & $0.07(0.11) \mathrm{b}$ & $0.16(0.24) \mathrm{d}$ \\
Control & & Total por hoja & \\
Citrolina & $2.65(0.27) \mathrm{a}$ & $2.52(0.13) \mathrm{a}$ & $3.46(0.19) \mathrm{a}$ \\
Cipermetrina & $0.91(0.27) \mathrm{c}$ & $0.22(0.12) \mathrm{b}$ & $4.97(0.19) \mathrm{a}$ \\
Citrolina + malatión & $0.64(0.27) \mathrm{c}$ & $0.19(0.12) \mathrm{b}$ & $1.96(0.19) \mathrm{b}$ \\
\hline
\end{tabular}

Medias con la misma letra en cada columna son estadísticamente iguales (Tukey, 0.05).

Cuadro 6. Control químico de la escama blanca del mango en árboles del cultivar 'Ataulfo'. Experimento 5. Atonalisco, Nayarit. 2006.

\begin{tabular}{lccc}
\hline \multirow{2}{*}{ Tratamiento } & \multicolumn{3}{c}{ Insectos por hoja $( \pm$ Error estándar) } \\
\cline { 2 - 4 } & Semana 0 & Semana 1 & Semana 2 \\
Control & $0.35(0.08) \mathrm{a}$ & $0.17(0.03) \mathrm{a}$ & $0.16(0.02) \mathrm{a}$ \\
Citrolina & $0.50(0.08) \mathrm{a}$ & $0.04(0.03) \mathrm{b}$ & $0.03(0.02) \mathrm{b}$ \\
Citrolina+malatión $\left(1.25 \mathrm{~mL} \mathrm{~L}^{-1}\right)$ & $0.43(0.08) \mathrm{a}$ & $0.15(0.03) \mathrm{a}$ & $0.01(0.02) \mathrm{b}$ \\
Citrolina+malatión $\left(2.5 \mathrm{~mL} \mathrm{~L}^{-1}\right)$ & $0.34(0.08) \mathrm{a}$ & $0.01(0.03) \mathrm{b}$ & $0.01(0.02) \mathrm{b}$ \\
& & Total por hoja & \\
Control & $2.01(0.28) \mathrm{a}$ & $0.88(0.20) \mathrm{a}$ & $2.36(0.20) \mathrm{a}$ \\
Citrolina & $1.51(0.28) \mathrm{a}$ & $0.51(0.20) \mathrm{ab}$ & $0.06(0.20) \mathrm{b}$ \\
Citrolina+malatión $\left(1.25 \mathrm{~mL} \mathrm{~L}^{-1}\right)$ & $1.95(0.28) \mathrm{a}$ & $1.00(0.20) \mathrm{a}$ & $0.04(0.02) \mathrm{b}$ \\
Citrolina+malatión $\left(2.5 \mathrm{~mL} \mathrm{~L}^{-1}\right)$ & $1.34(0.28) \mathrm{a}$ & $0.16(0.20) \mathrm{b}$ & $0.03(0.20) \mathrm{b}$ \\
\hline
\end{tabular}

Medias con la misma letra en cada columna son estadísticamente iguales (Tukey, 0.05).

cualquiera de las dos dosis de malatión $\left(1.11 \mathrm{~mL} \mathrm{~L}^{-1} \mathrm{o} 2.22\right.$ $\mathrm{mL} \mathrm{L}^{-1}$ ), fueron tratamientos efectivos para el control de la escama blanca del mango 'Ataulfo' en Atonalisco, Nayarit. En caso de aplicar estas mezclas en huertos productivos, se recomienda utilizar las dosis más bajas, para evitar un posible efecto negativo sobre las poblaciones de organismos benéficos.

\section{Experimento 6}

En este ensayo en que se probaron detergentes se detectó que antes de la aplicación de los productos la población de escama blanca (colonias y total de escamas) sólo fue ligeramente superior en los árboles destinados para la aspersión con detergente Roma ${ }^{\circledR}$ (Cuadro 7). Sin embargo, una, dos y tres semanas después de la aplicación, los promedios de colonias y del total de escamas de los tratamientos con detergente o con citrolina fueron estadísticamente más bajos que el testigo. (Cuadro 7).

El mejor control logrado con los detergentes Roma ${ }^{\circledR}$ $(75.5 \%)$ y Ariel ${ }^{\circledR}(55.3 \%)$ se obtuvieron a la primera semana después de la aplicación; el mejor control de citrolina se registró a la segunda semana (71.3\%). Tres semanas después de la aplicación de los tratamientos, los niveles de control fueron muy bajos; i. e., el control máximo con citrolina fue de apenas $51.9 \%$. Lo anterior indica que en general, los detergentes no alcanzaron los niveles altos de control semejantes al de algún insecticida tradicional (i.e., dimetoato del Experimento 2). Sin embargo, ante el interés por el uso de estos productos, se sugiere reforzar la investigación acerca de dosis y número de aplicaciones en mango de diversos 
Cuadro 7. Control químico de la escama blanca del mango en árboles del cultivar 'Ataulfo'. Experimento 6. Atonalisco, Nayarit. 2007.

\begin{tabular}{|c|c|c|c|c|}
\hline \multirow{2}{*}{ Producto } & \multicolumn{4}{|c|}{ Insectos por hoja ( \pm Error estándar) } \\
\hline & Semana 0 & Semana 1 & Semana 2 & Semana 3 \\
\hline & \multicolumn{4}{|c|}{ Colonias por hoja } \\
\hline Control & $0.17(0.1) b$ & $0.88(0.1) \mathrm{a}$ & $0.41(0.06) \mathrm{a}$ & $0.41(0.09) \mathrm{a}$ \\
\hline Ariel $\AA$ & $0.50(0.1) b$ & $0.42(0.1) b$ & $0.06(0.06) \mathrm{b}$ & $0.31(0.09) \mathrm{b}$ \\
\hline Citrolina & $0.28(0.1) \mathrm{ab}$ & $0.18(0.1) b$ & $0.06(0.06) \mathrm{b}$ & $0.12(0.09) \mathrm{c}$ \\
\hline \multirow[t]{2}{*}{ Roma ${ }^{\circledR}$} & $0.67(0.1) \mathrm{a}$ & $0.11(0.1) b$ & $0.11(0.06) b$ & $0.11(0.09) \mathrm{c}$ \\
\hline & \multicolumn{4}{|c|}{ Total por hoja } \\
\hline Control & $6.78(1.2) b$ & $8.68(0.7) \mathrm{a}$ & $4.81(0.4) \mathrm{a}$ & $3.08(0.3) \mathrm{a}$ \\
\hline Ariel $\mathbb{R}$ & $9.58(1.2) \mathrm{ab}$ & $3.88(0.7) b$ & $2.55(0.4) b$ & $1.91(0.3) b$ \\
\hline Roma ${ }^{\circledR}$ & $11.95(1.2) \mathrm{a}$ & $2.13(0.7) b$ & $1.55(0.4) b$ & $1.60(0.3) b$ \\
\hline Citrolina & $6.90(1.2) b$ & $4.10(0.7) b$ & $1.38(0.4) b$ & $1.48(0.3) \mathrm{b}$ \\
\hline
\end{tabular}

Medias con la misma letra en cada columna son estadísticamente iguales (Tukey, 0.05).

detergentes.

\section{CONCLUSIONES}

Con las mezclas de citrolina $\left(50 \mathrm{~mL} \mathrm{~L}^{-1}\right)+$ malatión (a dosis de $1.11 \mathrm{~mL} \mathrm{~L}^{-1}$ o $2.22 \mathrm{~mL} \mathrm{~L}^{-1}$ ) se obtuvo hasta 98.3 y 100 $\%$ de control de escamas, respectivamente. Hubo casos en los que las aspersiones de piriproxifeno $\left(0.3 \mathrm{~mL} \mathrm{~L}^{-1}\right)$ o dimetoato $\left(2.0 \mathrm{~mL} \mathrm{~L}^{-1}\right)$ lograron controlar hasta $100 \%$ de escamas. Cuando se aplicó el aceite mineral (citrolina) en dosis de $50 \mathrm{~mL} \mathrm{~L}^{-1}$ o $75 \mathrm{~mL} \mathrm{~L}^{-1}$, el máximo control de escamas fue de 91.3 y $97.5 \%$, respectivamente. Con los detergentes comerciales Roma ${ }^{\circledR}$ y Ariel ${ }^{\circledR}\left(20 \mathrm{~g} \mathrm{~L}^{-1}\right)$ los mejores controles que se lograron fueron durante la primera semana con 75.5 y $55.3 \%$, respectivamente. Los resultados con cipermetrina a 0.24 y $0.36 \mathrm{~mL} \mathrm{~L}^{-1}$ y con el producto a base de ajo fueron inconsistentes. Con cipermetrina el control más alto de escamas que se alcanzó fue de $92.5 \%$ y con el concentrado de ajo fue de $57.13 \%$. Estos resultados ofrecen opciones relativamente seguras para el control de la escama blanca del mango, como es el caso del uso de citrolina, aunque es necesario validar estos resultados con más investigación al respecto.

\section{AGRADECIMIENTOS}

Al Fondo Sectorial SAGARPA-CONACYT, por el financiamiento otorgado para esta investigación a través de los proyectos 2006-48122 y 2012-171759.

\section{BIBLIOGRAFÍA}

Arias De L M, A Jines C, C Carrera, N Bustos N, P Plúas M, K Gutiérrez (2004) Biología, dinámica poblacional, muestreo, nivel de daño y alternativas para el manejo de Aulacaspis tubercularis en mango de exportación. Instituto Nacional Autónomo de
Investigaciones Agropecuarias. Folleto Técnico No. 56. Guayaquil, Ecuador. 19 p.

Butler Jr G D, T J Henneberry, P A Stansly, D J Schuster (1993) Insecticidal effects of selected soaps, oils and detergents on the sweetpotato whitefly: (Homoptera: Aleyrodidae). Florida Entomol. 76:161-167.

Curkovic T, J E Araya (2004) Acaricidal action of two detergents against Panonychus ulmi (Koch) and Panonychus citri (McGregor) (Acarina: Tetranychidae) in the laboratory. Crop Prot. 23:731733.

García-Álvarez N C, M A Urías-López, J A González-Carrillo (2008) Distribución geográfica de la escama blanca Aulacaspis tu bercularis Newstead (Hemiptera: Diaspididae) del mango en Nayarit. Ent. Mex. 7:640-645.

González-Carrillo J A, M A Urías-López, N C García-Álvarez (2008) Fluctuación poblacional de la escama blanca, Aulacaspis tu bercularis Newstead (Hemiptera: Diaspididae) del mango en Nayarit, México. Ent. Mex. 7:646-651.

Le Lagadec M D, C E Louw, C Labushagne (2009) The control of scale insects and mealybugs on mangoes in South Africa using neonicotenoids: A review of experimental work from 2001 to 2005 Acta Hort. 820:549-557.

Pless C D, D E Deyton, C E Sams (1995) Control of San Jose scale, terrapin scale, and European red mite on dormant fruit trees with soybean oil. HortScience 95:94-97.

SAS (2000) SAS/STAT User's Guide. Version 8. SAS Institute Inc. Carey, NC.

Rae D J, W G Liang, D M Watson, G A Beattie, M D Huang (1997) Evaluation of petroleum spray oils for control of the Asian citrus psylla, Diaphorina citri (Kuwayama) Hemiptera: Psyllidae), in China Int. J. Pest Manage. 43:71-75.

Rebek E J, S S Clifford (2003) Effects of pesticide applications on the euonymus scale (Homoptera: Diaspididae) and its parasitoid, Encarsia citrina (Hymenoptera: Aphelinidae). J. Econ. Entomol. 96:446-452.

SAGARPA-CESAVENAY (2007) Superficie de Mango, Volumen de Producción y Rendimiento Promedio por Superficie y por Variedad. Subdirección Agropecuaria. Programa de Fomento Agrícola y Programa de Sanidad Vegetal. Delegación Estatal de Nayarit. $10 \mathrm{p}$

Smith E H, G W Pearce, (1948). The mode of action of petroleum oils as ovicides. J. Econ. Entomol. 41:173-180.

Stadler T, M Buteller (2009). Modes of entry of petroleum distilled sprayoils into insects: a review. Bull. Insectol. 62:169-177.

Thakur A K, S F. Hameed (1980). Toxicity of some organophosphorus insecticides to the crawlers of San Jose scale Quadraspidiotus perniciosus Comst. Pest. Bombay 14:10-12 
Urías L M A (2006) Principales plagas del mango en Nayarit. In: El Cultivo del Mango: Principios y Tecnología de Producción. V Vázquez V, M H Pérez B (eds). Instituto Nacional de Investigaciones Forestales Agrícolas y Pecuarias. Santiago Ixcuintla, Nay. pp:211-234.

Urías-López M A, R Flores-Canales (2005) La "escama blanca", Aulacaspis tubercularis Newstead (Homóptera: Diaspididae) una nueva plaga del mango: Fluctuación poblacional y anotaciones biológicas. Ent. Mex. 4:579-584.
Urías-López M A, J A Osuna-García, V Vázquez-Valdivia, M H PérezBarraza (2010) Fluctuación poblacional y distribución de la escama blanca del mango (Aulacaspis tubercularis Newstead) en Nayarit, México. Rev. Chapingo S. Hort. 16:77-82.

Wakgari W, J Giliomee (2001) Effects of some conventional insecticides and insect growth regulators on different phenological stages of the white wax scale, Ceroplastes destructor Newstead (Hemiptera: Coccidae), and its primary parasitoid, Aprostocetus ceroplastae (Girault) (Hymenoptera: Eulophidae). Int. J. Pest Manage. 47:179-184. 